\title{
Problemas y perspectivas en torno a las concepciones humanas conjeturales en la terapia cognitivo-conductual
}

\author{
PROBLEMS AND PERSPECTIVES CONCERNING THE HUMAN CONJECTURAL CONCEPTIONS IN COGNITIVE \\ - BEHAVIORAL THERAPY
}

Dr. Niklas Bornhauser (nbornhauser@unab.cl) Escuela de Psicología, Universidad Andrés Bello (Santiago, Chile)

Dr. Rudi Wagner (wagner01@ph-ludwigsburg.de) Ludwigsburg University of Education International (Ludwigsburg, Alemania)

\begin{abstract}
Nowadays psychology as a scientific discourse and a positive practice finds itself in an epistemologically critical situation. The analysis of the actual state of the academic discussion in cognitive-behavioural psychology, the most representative and widespread theoretical-practical trend in European nations, reveals that it frequently is misunderstood as a exclusively technical proceeding, an amount of deficiently articulated operatory interventions, alienated from its underlying anthropological assumptions. This paper proposes to exam how far the gap between theoretical reflection and effective practice, a cleavage frequently confirmed, may be reduced discussing the concept of Menschenbildannahmen, an idea firmly rooted in anthropological thinking.
\end{abstract}

Key words: psychology, anthropology, theory, practice

\section{Resumen}

Actualmente, la psicología en tanto discurso científico y práctica positiva se encuentra en un estado epistemológicamente crítico. El análisis del estado actual de la discusión académica en la psicología cognitivo-conductual, la corriente teórico-práctica más representativa y más difundida en los estados europeos, devela que ésta frecuentemente es malentendida como un mero procedimiento técnico, un conjunto precariamente articulado de intervenciones operatorias, desentendidas de sus supuestos antropológicos subyacentes. Se propone examinar en qué medida la brecha entre reflexión teórica y práctica efectiva, una escisión ampliamente constatada, puede ser aminorada sometiendo a discusión el concepto de las Menschenbildannahmen, de extensa raigambre en el pensamiento antropológico.

Palabras clave: psicología, antropología, teoría, práctica 


\section{Planteamiento del problema}

Desde los trabajos de Groeben y Michalski, la hipótesis que las teorías científicas acerca del hombre contienen determinadas representaciones antropológicas básicas (qua Menschenbildannahmen) (1), ha sido profusamente recibida. De este modo, Barsch (2000), en su trabajo centrado en la secularización y pluralización de las representaciones de la naturaleza humana, ha enfatizado que su naturaleza preconceptual las predispone para que se sustraigan de toda problematización y discusión reflexiva. En el contexto de la teoría analítica de las ciencias, Jaeggi ha trabajado este problema principalmente bajo la perspectiva de las llamadas non-statement views. De acuerdo a este punto de vista, las formaciones teóricas no pueden ser reducidas a un número o sistema de afirmaciones o enunciados -tal como postula la concepción clásica de enunciados de teorías, la llamada statement view-, sino que más bien han de ser pensadas como complejos constructos discursivos del tipo conceptual y como tales resultan inmunes frente a la experiencia y, por lo tanto, a la falsificación. Los supuestos básicos «latentes» acerca del hombre se encuentran ubicados en este núcleo teórico de difícil aprehensión y, por ende, no pueden ser revisadas empíricamente sin más. Al no ser accesibles directamente a la conciencia crítica y sustraerse empecinadamente a la problematización teórica, dichos supuestos tienen una influencia, larvada y encubierta pero considerable, sobre la metodología característica y las tecnologías derivadas de aquellas teorías.

En el ámbito psicoterapéutico, los supuestos antropológicos preconceptuales obtienen una relevancia particular: la descripción, el diagnóstico, la comprensión y el tratamiento de alteraciones de la psyché, debido al estatuto hipotético del objeto en cuestión, epistemológicamente inseguro, efímero, más bien débil, siempre implica una representación -no siempre admitida- de qué es "el hombre», de cómo funciona y de cómo puede ser influenciado por sí mismo o por otros. Supuestos antropológicos preconceptuales contenidos en toda teoría psicoterapéutica, influyen tanto sobre la percepción -y, por lo tanto, sobre el diagnóstico y el tratamiento subsecuente- de determinadas alteraciones (comprobado empíricamente, por ejemplo, por Faller, Wagner y Weiß 2000), así como sobre la construcción -social- de la realidad (Berger y Luckmann 1979). Empero, a pesar de la evidente relevancia del tema, resulta sorprendente que a la discusión científica de los supuestos antropológicos preconceptuales asociadas a las diferentes prácticas psicoterapéuticas se le haya dedicado más bien poca atención. Una posible razón para ello puede ser visto en que los supuestos antropológicos preconceptuales, y ello precisamente caracteriza y subraya su naturaleza esquiva y problemática, rara vez son formulados y tematizados explícitamente y, de esta manera, suelen seguir operando en un nivel que ha sido distinguido como ideológico.

El examen crítico-reflexivo de las principales corrientes o tendencias psicoterapéuticas bajo la perspectiva de los supuestos antropológicos preconceptuales subyacentes, exige la construcción previa de un punto de vista, Standpunkt, que facilite el análisis histórico-crítico de una situación. Recién a partir de una consideración histórica de las posturas examinadas, pueden ser debidamente reconocidas y analizadas las particularidades y las restricciones de determinadas direcciones terapéuticas particulares (Wagner), detallando su ubicación relativa en el enmarañado entramado teórico que describe la situación actual en psicoterapia. Desde luego, que esta discusión con la historia de la psicoterapia implica la ejecución de un complejo proceso de análisis que, si bien contiene dimensiones o problemáticas compartidas o supraordenadas, debido a la especificidad de los factores a considerar, ha de ser efectuado principalmente para cada escuela o corriente por separado. A continuación, en primer lugar se describirá el estado actual de la discusión a propósito de las repercusiones de los supuestos antropológicos preconceptuales en las 
llamadas teorías conductuales, para en un segundo momento someter a examen las respuestas generadas hasta el momento desde su mismo interior al problema de los supuestos antropológicos preconceptuales.

\section{Los supuestos antropológicos implícitos en la terapia conductual: del objeto reductivo hacia el sujeto reflexivo}

Al examinar en detalle las diferentes corrientes terapéuticas bajo la perspectiva de los supuestos antropológicos preconceptuales, la forma contemporánea derivada de la terapia conductual, la llamada terapia cognitivo-conductual, destaca por el hecho de implicar dos conceptos de sujeto altamente discrepantes, tanto en lo que se refiere a la concepción de hombre más general, como en lo relacionado con la estructuración y el funcionamiento de su psiquismo en particular. Nuestra hipótesis es que dicha situación, a saber, la coexistencia simultánea de dos nociones de sujeto diferidas, descritas por Kazdin (1978) y Schorr (1984), es el resultado de los supuestos antropológicos preconceptuales subyacentes, en ocasiones altamente opuestos y hasta incompatibles.

1) La imagen del hombre de la terapia conductual temprana

La terapia conductual se desarrolló a principios del siglo XX al interior del paradigma behaviorista constituyéndose en oposición consciente e intencionada a los procedimientos interpretativos, altamente especulativos, característico de las concepciones psicoterapéuticas predominantes en aquel entonces. Sobre el fundamento de la concepción -y comprensión- behaviorista de ciencia se desarrollaron numerosas teorías y métodos, dispuestas a prescindir de las especulaciones y teorizaciones de incierto valor objetivo. De ahí en adelante, sólo la conducta "abierta», sobre cuya presencia o ausencia cuantificable se pudiera decidir con -relativa- objetividad, debía ser objeto de la construcción de teorías científicas, dotándolas así idealmente de mayor consistencia y solidez conceptual. En nuestra opinión, la concepción de una conducta presuntamente abierta, descuida justamente el hecho que la conducta no yace simplemente ahí, en espera de ser captada por la percepción de un sujeto cognoscente, sino que se encuentra oculta, verborgen, y ha de ser des-cubierta mediante un movimiento de Offenlegung similar al aletheia descrito por Heidegger.

Las primeras teorías behavioristas implicaban un concepto de hombre (2) excesivamente reductivo: los supuestos antropológicos centrales del behaviorismo -entre ellos, el modelamiento del hombre como un objeto (más), que se limita a reaccionar de manera previsible a estímulos provenientes del exterior (modelo black-box)- proporcionan los cimientos sobre los cuales se articularía todo su intrincado edificio teórico. Este énfasis en los aspectos palpables (3), aprehensibles mediante la experiencia sensible, conllevó paralelamente un descuido -relativo- de posibles procesos mentales arraigados en el acontecer y vivenciar psíquico del hombre, tales como pensamiento, percepción, etc. Watson, en su artículo Psychology as the behaviourist views it enfatizaba de manera ejemplar que el behaviorismo consideraba que los procesos de la mente propiamente tales -en otras palabras, que Watson probablemente desaprobaría, los procesos mentales «en sí»- no son directamente accesibles a un análisis científico, por lo cual se debía prescindir de toda información acerca de semejantes estados nebulosos y evanescentes como eventual fuente de datos para la ulterior investigación científica. Skinner en 1953, en un texto paradigmático titulado Science and human behaviour se pronunciaría de manera parecida, reafirmando la posición adoptada por Watson. Estos escritos comparten con sus elaboraciones posteriores el hecho que al paciente, en el marco de las teorías conductuales, le es asignado un rol pasivo, objetal, que lo condena a reaccionar de manera previsible a determinados estímulos exteriores, sometiéndolo al albedrío de factores ajenos, provenientes desde el 
exterior. El ser humano, tal como lo postula el conductismo, es modelado de manera paralela y equivalente a como sucede con organismos cognitivamente limitados -aparentemente carentes de las facultades conscientes y volitivas que caracterizan y distinguen al hombre-, ya que el comportamiento humano es pensado en estricta analogía con el comportamiento de los animales. Bandura (1986) en este contexto habla de un determinismo unidireccional, ya que la conducta humana, representada como efecto o resultado de una serie de operaciones precedentes, en el marco de semejante concepción de hombre, es dirigida y dictada unilateralmente por condiciones y factores ambientales.

Semejante concepción determinista, marcada por el paradigma mecanicista temprano, inspirado en las investigaciones científicas de Galileo, Huygens y Boyle, enunciado finalmente por René Descartes, hoy en día es defendida únicamente por aquella vertiente del conductismo que se conoce como conductismo radical o duro. Westmeyer, en un trabajo de 1984, hace notar que es posible distinguir diferentes tipos de conductismo aplicando ciertos criterios de desglose crecidos históricamente. La clasificación más difundida de manera didáctica separa tres posiciones fundamentales: primero, el conductismo metafísico, segundo, la versión radical o analítica y, finalmente, el conductismo metodológico. Mientras que las primeras dos formas rechazan a priori la existencia de acontecimientos psíquicos, el conductismo metodológico, en cambio, debido a sus propias restricciones internas, se abstiene de emitir opinión alguna al respecto, ya que se define principalmente mediante un procedimiento metodológico determinado y así elude la pregunta por la concepción de hombre implicada. Sin embargo, también esta aproximación, de manera análoga como sucede con las dos primeras, conduce a un estrechamiento de la visión de las cosas, tal como ha sido retratado en el contexto de la interacción objeto-método. Entidades o sucesos como los eventuales procesos cognitivo-emocionales del paciente, sus propias teorías (subjetivas, valga la redundancia), sus planes, ideas y metas, su libre intencionalidad no son percibidas ni problematizadas en el contexto del modelo black-box. Groeben (1986) a propósito de esta discusión habla de la "pervertibilidad tecnológica», una amenaza inherente -aunque no exclusivamente- a toda teoría científica que parta de un concepto de hombre reduccionista.

La elección de los procedimientos psicoterapéuticos indicados, aplicando únicamente el criterio de su efectividad empírica, descuidando, en cambio, sus supuestos epistemológicos y sus implicaciones éticas, sin duda puede ser calificada como utilitarista. Este enfoque, que goza de particular popularidad entre las aproximaciones pragmáticas y utilitaristas de la teoría conductual, encierra el peligro de privilegiar una racionalidad orientada a fines en desmedro de aspectos pertenecientes a la racionalidad valórica (Weber) y así producir efectos secundarios negativos y no-intencionados. En las terapias conductuales de los primeros tiempos, al igual que en las terapias contemporáneas de corte ortodoxo o radical, los principios fundamentales del condicionamiento clásico y operante fueron aplicados irreflexivamente a gran número de pacientes sin considerar la dimensión ética de semejante proceder. Szasz ha investigado numerosos casos en los cuales se efectuaron tratamientos aversivos dirigidos hacia la extinción de conductas "no deseadas», aplicando estímulos no solamente desagradables sino incluso dolorosos, tales como inyecciones de sustancias heméticas, shocks eléctricos, etc. con tal de lograr una reducción de las conductas indeseadas y facilitar una adaptación lograda a la norma. Braunstein ha advertido acerca de los peligros que corre la psicología acrítica, pragmática, de convertirse en el receptor y ejecutor de un determinado encargo o demanda social, formulada por los estamentos dominantes de cada sociedad, y que, desde luego, implica ciertas ideas a propósito del concepto de normalidad asociado. Raymond informa del tratamiento de pacientes con desviaciones sexuales mediante inyecciones de apomorfina, que gatillaba en los afectados 
reacciones de asco y malestar. Feldman y McCulloch (1971) reportan gran número de casos en los cuales se sometía a «terapia» a pacientes homosexuales aplicándoles shocks eléctricos, acoplados a la presentación de imágenes de hombres desnudos, como estímulos aversivos. Estos ejemplos solamente han de ilustrar los alcances que pueden adquirir las derivaciones tecnológicas de determinadas teorías científicas si se descuida o suprime la discusión de su respectivo fundamento antropológico. Dicho fundamento, en la medida en que pone el énfasis no solamente en el ser humano sino en la revisión necesaria de los supuestos y prejuicios necesariamente valóricos- que condicionan su representación, siempre implica la demanda de una reflexión ética entendida como la pregunta por cómo se justifica racionalmente un sistema moral y cómo se ha de aplicar posteriormente a los distintos ámbitos de la vida personal y social. La mentada oposición entre racionalidad valórica y racionalidad orientada a fines puede ser pensada como analogía de la oposición entre una psicología centrada en la revisión de sus supuestos antropológicos y una psicología orientada a un sistema de fines individuales, mantenido conscientemente por un agente, que contempla a los objetos externos como medios o como obstáculos para estos fines. A nuestro parecer, no es que el modelo blackbox, que establece como características centrales del paciente la reactividad y el control exógeno subsecuente por medio de la oscilación de ciertos factores del ambiente, necesariamente conlleve consecuencias fatales y nefastas para la humanidad. Más bien, lo que nos interesa subrayar es cómo la falta de consideración de un supuesto antropológico, respectivamente, la falta de análisis de su proceso de generación, expone la praxis científica a los riesgos anteriormente mencionados.

No obstante, por muy fundada que pueda parecer esta crítica al concepto de hombre postulado en su momento por el conductismo temprano, ateniéndose a los resultados preliminares del Positivismusstreit, si realmente se desea analizar la situación en perspectiva, se ha de someter a examen la situación histórica y las condiciones materiales en las cuales se han conformado dichas estructuras de investigación. Ha sido uno de los méritos indiscutidos del llamado debate positivista, centrado en la discusión sobre los métodos y los juicios valóricos en ciencias sociales, el enfatizar que las formulaciones teóricas en ciencia conllevan necesariamente juicios de valor. Al contextualizar históricamente los resultados científicos e inscribirlos en un determinado marco cognoscitivo, no se hace otra cosa sino intentar aprehender la historicidad efectual de un concepto y paradigma determinado y con ello develar y comprender la lógica de su devenir y sus futuras posibilidades.

De dicha historización se espera obtener como resultado una visión más matizada, más diferenciada de la problemática de los supuestos antropológicos preconceptuales, ya que también -y sobre todo- en la actualidad hay que discutir la relevancia de estas teorías y sus procedimientos derivados para el desarrollo de la ciencia psicológica y para la formulación de nuevas estrategias de tratamiento. Tanto un rotundo rechazo apriorístico de teorías y procedimientos derivados de la teoría conductual como la sustitución mecánica por su contrario indudablemente son reacciones inmaduras e inadecuadas, que, más que producir un cambio cualitativo real o de provocar la transformación efectiva deseada, perpetúan un determinado status quo mediante el mecanismo de la inversión simple. Los sorprendentes resultados clínicos de determinados procedimientos terapéuticos basados en las teorías en cuestión -por ejemplo, el tratamiento de la angustia (Margraf y Schneider 2000) o de los síntomas obsesivos (Reinecker 1998)-, son un poderoso argumento a favor de la revisión pormenorizada y desprejuiciada de los planteamientos cognitivoconductuales al respecto (4). 
Sin embargo, ante el trasfondo de las observaciones precedentes, sí parece criticable la sobregeneralización irreflexiva de las regularidades conductuales y su extensión, falta de diferenciación, a la totalidad de las conductas humanas, tal como la exigían las teorías conductuales tempranas sobre la base de la demanda de ubiquidad. Los análisis de Foucault acerca de la articulación entre saber y poder han demostrado recientemente cómo semejantes pretensiones de universalidad han de ser interpretados como aspiraciones totalitarias de poder, derivadas de una «voluntad de poder» (Nietzsche) destructiva e insaciable. A propósito de esto último uno no puede dejar de insistir en que la verdad no es ajena al poder, no está fuera de él ni sin el poder. La verdad, en lugar de situarse en el recóndito y remoto mundo de las ideas, más allá de la materialidad y tosquedad chocantes de las apariencias, está de este lado de las cosas, es de este mundo. Y, en vez de preexistir a la investigación desinteresada y abnegada, aguardando pacientemente ser descubierta por la mirada de algún investigador astuto, está siendo producida aquí y ahora gracias a múltiples coacciones e imposiciones. Es así como cada sociedad tiene su régimen de verdad, su «política general de la verdad», es decir, su administración reguladora, los mecanismos y las instancias que permiten distinguir los enunciados falsos y verdaderos, de los tipos de discursos que ella acoge y hace funcionar como verdaderos, por un lado, y de aquellos que excluye, prohíbe y sanciona, declarándolos como falsos. Esta política combina entre sí, por ejemplo, las maneras de reforzar y sancionar unos y otros, las modalidades técnicas que son establecidas, jerarquizadas y valorizados para la obtención de la verdad y el estatuto de aquellos encargados de dictaminar qué es lo que funciona como verdadero sobre las espaldas de aquello que no lo hace. En sociedades como las nuestras, independientemente de que las designemos como postindustriales, neoliberales, tardocapitalistas, etc., la economía política de la verdad, si resumimos el argumento foucaultiano, está caracterizada por 5 rasgos: la verdad está centrada en la forma del discurso científico y en la instituciones que lo producen; está sometida a una constante incitación económica y política; es objeto bajo formas diversas de una inmensa difusión y consumo; es producida y transmitida bajo el control no exclusivo pero si dominante de algunos grandes aparatos políticos o económicos (universidad, ejército, escritura); en fin, es el núcleo de la cuestión de todo un debate político y de todo un enfrentamiento social. A su vez, el debate científico al interior de la llamada "comunidad lingüística», como ha argumentado Habermas (1997), ha de estar precedido por la definición previa de los lugares y las posiciones desde las cuales se manifiestan los sujetos hablantes implicados y la clarificación racional de las posibles significaciones atribuibles a ciertos conceptos fundamentales. Solamente tras asegurar el que sean respetadas determinadas reglas elementales, cuyo cumplimiento constituye las condiciones imprescindibles para asegurar el debate democrático y abierto, pueden ser relativizados los enunciados y las declaraciones de los sujetos comunicantes entre sí ante el trasfondo de un mundo vivencial lingüísticamente articulado (5).

\section{2) El hombre como actuante activo en el enfoque del selfmanagement}

Como reacción histórica a las palmarias críticas del modelo black-box sostenido por el conductismo ortodoxo, en el contexto del llamado "giro cognitivo" que experimentaba la psicología como discurso científico, fue desarrollado un concepto de hombre que enfatizaba especialmente sus aspectos «interiores», es decir, sus procesos mentales, especialmente su pensar y sentir. Una descripción exhaustiva y detallada de este vuelco cognoscitivo en cuanto a la representación del subjetivo objeto de estudio de la psicología se encuentra, por ejemplo, en los trabajos de Reinecker y Seiwert. En el caso del conductismo, con respecto a esta forma reflexiva en el pensamiento de la psicología, ha de ser destacado sobre todo el aporte de $\mathrm{H}$. Kanfer $(1970,1979)$, quien ha sido calificado como el responsable de abrir el hermetismo de la black-box conductista. Este nuevo concepto de hombre, centrado en la consideración de sus facultades y funciones 
interiores, se convertiría en el fundamento para el enfoque (6) del selfmanagement, cuyos aspectos principales se expondrán resumidamente a continuación.

En el marco del enfoque del selfmanagement, el hombre, que en los tiempos del conductismo temprano había sido despojado de su deseo y voluntad, ahora recupera sus facultades activas, su capacidad de expresarse y manifestarse mediante comportamientos y enunciados. De acuerdo a este desplazamiento del centro de gravedad de lo humano desde la conducta reactiva y el determinismo unidireccional hacia la interioridad complejamente estructurada y la actividad reflexiva, las construcciones-de-mundo y de-sí devienen entidades centrales, que han de ser problematizadas como tales en el tratamiento psicoterapéutico. El selfmanagement de acuerdo a los autores citados alude a la capacidad del hombre no solamente de determinar y fijar metas desde sí, sino de desarrollar a partir de estas metas planes comportamentales secuenciados jerárquicamente ordenados y de conducirse de acuerdo a sus metas y planes establecidos; es decir, se revaloran el deseo y la voluntad del hombre en tanto fuente activa de sus acciones y comportamientos. Por lo tanto, una terapia de selfmanagement plantea como meta terapéutica ayudar al cliente a adquirir una capacidad incrementada de dirigir conscientemente su proceso de toma de decisiones y de conducir las acciones derivadas, o sea, de dirigir conscientemente su vida de acuerdo a sus ideales y valores libremente elegidos. El cliente, bajo esta perspectiva ha de convertirse en «experto de sus problemas» (Lieb y Lutz 1992) y con este incremento de su competencia y experticia desestabiliza la tradicional jerarquía, establecida entre el terapeuta, omnisapiente y autónomo, por un lado, y el paciente, ignorante y sufriente, dependiente de la voluntad del primero, por el otro. En esta reconceptualización de la relación terapeuta-paciente se muestra un concepto de hombre, según el cual el individuo en principio es capaz de aprender determinadas estrategias de autorregulación y de incrementar, acentuar y regular su grado de autodeterminación, autonomía y libertad personal. En base a estos conceptos se podría hablar de un concepto de hombre heredado de la filosofía ilustrada.

A diferencia de los supuestos antropológicos preconceptuales mecanicistas y reduccionistas de las teorías de la terapia conductual temprana, en esta acepción de hombre consciente e intencional se destacan aspectos positivos del ser-hombre, que no han de estar presentes desde siempre, pero cuya realización se supone como posible y es connotada positivamente. En el concepto de hombre del selfmanagement son los aspectos racionales los que son destacados explícitamente al colocar las capacidades de reflexión y de comunicación en el centro de las observaciones en terapia. Este giro reflexivo, desde otro lugar, puede ser interpretado como un retorno après-coûp a los principios racionalistas impulsados por Descartes con tal de fundar y propugnar la noción del cogito cartesiano. Se puede hablar, por lo tanto, de una especie de recreación de un sujeto definido por la mentalidad moderna temprana, con la exaltación imaginaria de las facultades de la razón y la pérdida concomitante de la experiencia conatural de la naturaleza, un tipo de subjetividad que desmiente las críticas recientes a la supuesta autonomía y coherencia del sujeto moderno.

La capacidad del hombre de reflexionar acerca de la generación del sentido y el rumbo de su vida, el sentido del Dasein, y de comportarse reflexivamente con respecto a sus determinaciones y acciones resultantes recién es considerado desde esta perspectiva. Terapeuta y paciente -a diferencia de muchas otras vertientes o corrientes teóricas- son modelados en una especie de paralelismo estructural: en principio, ambos disponen de las mismas capacidades o posibilidades evolutivas, un potencial creativo que espera ser descubierto y desarrollado. 
Este concepto "positivo» de hombre ha producido sus propios sedimentos terapéuticos concretos, como demuestra el impacto y la difusión de los trabajos de Ellis, Beck y Wolpe y Lazarus. Las psicoterapias que se adscriben a semejante concepto de hombre, a diferencia de las terapias conductuales "rústicas», no se conforman con contabilizar y analizar comportamientos externos, sino, de manera reforzada, se concentran en la problematización de cogniciones y emociones del paciente. Las teorías subjetivas del paciente obtienen un rol central bajo el aspecto de la construcción del mundo, el principio de los procesos ocultos o velados se vuelve más y más importante.

Ante el trasfondo de este concepto de hombre, caracterizado por el énfasis en sus capacidades cognitivas, un aspecto importante de la terapia conductual deviene el no solamente informar al paciente acerca de su "alteración» o «enfermedad», sino el promover la construcción activa de un modelo psíquico correspondiente. El paciente, lejos de verse reducido a una entidad clínica vacía y alienada, al participar efectivamente del proceso de diagnóstico y de comprensión de su malestar, queda en condiciones de estudiar y de explorar cognitivamente su cuadro sintomatológico, su génesis y devenir, su pronóstico y los tratamientos y las intervenciones recomendadas y de ir convirtiéndose en perito y conocedor experto de su enfermedad. Consecuentemente, las metas terapéuticas del tratamiento psicológico ya no se siguen automáticamente de los síntomas diagnosticados ni son decretados autoritariamente desde el exterior por el aparato psicoterapéutico -el proceso de clarificación y diferenciación de las metas respectivas en el enfoque del selfmanagement representa una fase propia de la terapia y, al tratar con el deseo, el malestar, el sufrimiento y las motivaciones del paciente, adquiere un rol fundamental para su desarrollo ulterior. En esta fase inaugural del proceso terapéutico, terapeuta y paciente mediante una especie de diálogo socrático determinan conjuntamente qué metas han de ser alcanzadas mediante la terapia, de qué medios y recursos se dispone y cómo se proyecta en detalle el camino hacia su realización.

Desde luego, para este concepto de hombre al igual que para los anteriores ha de ser destacado su alcance limitado y sus salvedades correspondientes, ya que no todas las actividades y cualidades del hombre pueden ser descritas y explicadas mediante este modelo. Muchos comportamientos transcurren automáticamente, siguiendo una lógica propia, al margen de la conciencia, es decir, con una escasa participación de los controles cognitivos. Miller, Galanter y Pribham ya en 1960 habían constatado que la explicación y comprensión de la conducta humana, debido a su carácter altamente compuesto, ha de distinguir entre varios niveles diferenciados de conciencia y, por consiguiente, de control. Muchas formas de reaccionar se han ido configurando y endureciendo en el transcurso de la vida y no son accesibles a un control consciente. Por lo visto, también este concepto de hombre posee un ámbito de validez acotado y restringido, limitado por sus restricciones epistemológicas inherentes.

\section{Problemas de los supuestos antropológicos implícitos discrepantes en la terapia cognitivo-conductual}

Actualmente, tras decenios de reflexión y de experimentación, la terapia conductual (ya) no se define como una escuela hermética, cerrada, sino como una orientación terapéutica abierta y dispuesta al diálogo con otras tendencias o corrientes. Las diferentes definiciones que circulan por el espacio discursivo sostenido por la psicología comparten entre sí la orientación pragmática según los resultados empíricos obtenidos mediante la investigación científica. La denominación compuesta "terapia cognitivo-conductual», como ha sido expuesto por Fiedler (1997) y Mahoney (1997), ilustra que bajo este rótulo se reúnen dialécticamente procedimientos conductuales tempranos y métodos cognitivos más recientes, que se remontan a 
constelaciones problemáticas y condiciones histórico-materiales igualmente diferentes, que a su vez implican supuestos antropológicos preconceptuales sustancialmente diferentes. Métodos de flooding, control de estímulos y procedimientos operantes, por ejemplo, tienen su origen en la terapia conductual temprana e implican la aceptación del comentado modelo de black-box del hombre; procedimientos cognitivos como el diálogo socrático, técnicas discursivas racionales, goal-attainment-scaling, por otra parte, implican el concepto de hombre del enfoque del selfmanagement, bajo el cual el paciente, a diferencia del abordaje anterior, es pensado como alguien capaz de comportarse activa y selectivamente hacia los objetos y estímulos, tanto externos como internos, que construye una representación de sí y del mundo y se comporta de acuerdo a estas representaciones.

Por supuesto, que dos supuestos antropológicos preconceptuales tan discrepantes, que coexisten simultáneamente en un mismo espacio discursivo, frecuentemente pueden conducir a problemas teóricoprácticos espinosos. A continuación, algunos de estos problemas a nuestro parecer decisivos para el rumbo de la psicoterapia asociada a las teorías cognitivo-conductuales, serán sometidos a un análisis tan pormenorizado como sea posible en este contexto.

1) Problemas de imagen de la terapia cognitivo-conductual

La imagen temprana del hombre, propuesta y promovida por el behaviorismo de primera hora, cuyos principios y supuestos marcaron decisivamente a la terapia conductual en sus comienzos, fue criticada desde las más diversas posiciones. En la situación contemporánea, en la cual la terapia cognitivo-conductual ha evolucionado hacia el enfoque del selfmanagement, esta crítica, enunciada en los comienzos del conductismo de primera hora, solamente se aplica a una fracción reducida al interior del amplio y variado espectro de la terapia cognitiva contemporánea. Y sin embargo, a pesar del evidente desarrollo teórico propulsado por la revisión crítica de los propios fundamentos, la disposición negativa -en su mayoría excesivamente rotunda y falta de matices- que ha resultado de estas raíces behavioristas, aun sigue con vida. Este lamentable pre-juicio sigue siendo mantenido, entre otras cosas, por la práctica clínica de ciertos psicoterapeutas, que han adquirido su saber sobre la terapia conductual antes del giro cognitivo. El problema de imagen que aqueja a la terapia cognitivo-conductual, empero, no puede ser explicado aludiendo únicamente a la predisposición sesgada y la hostilidad del mundo exterior, sino que, naturalmente, también se relaciona con la autorrepresentación de los terapeutas conductuales y su posicionamiento subjetivo con respecto al discurso que (so)portan.

Una segunda dificultad, relacionada con la problemática anteriormente ilustrada, consiste en la falta de aplicación sistemática de procedimientos cognitivo-conductuales, a pesar de su eficiencia empíricamente probada. Así, por ejemplo, muchos terapeutas formulan sus recelos con respecto a la aplicación de técnicas de intervención basadas en el modelo black-box, ya que dicen tener problemas con la representación excesivamente reductiva del hombre que les subyace. Esta es una razón central, por la cual dichas operaciones (por ejemplo, procedimientos de confrontación o técnicas operantes), a pesar de su comprobada efectividad experimental, sólo se suelen aplicar rara vez, incluso en los tratamientos denominados cognitivo-conductuales. Incluso en el caso de los trastornos de ansiedad, es decir, aquel grupo de las alteraciones psíquicas en las cuales intervenciones cognitivo-conductuales -como, por ejemplo, los diversos procedimientos basados en el principio de la confrontación- han probado su efectividad por encima de otras aproximaciones terapéuticas, tal como demuestran las investigaciones realizadas por 
Margraf (2000), solamente un $1 \%$ de pacientes a los cuales les había sido diagnosticado un trastorno de ansiedad fueron tratados con procedimientos conductuales.

Una posible explicación para esta discrepancia entre el discurso oficial de la psicología cognitivo-conductual y la práctica clínica efectiva podría ser que la no-aplicación de procedimientos efectivos está condicionada sobre todo por la falta de difusión del saber cognitivo-conductual, una merma causada por las deficitarias y tendenciosas condiciones infraestructurales anteriormente aludidas. En contra de esta opinión, el trabajo de Wittchen (1996) demuestra que la falta de conocimientos y la falta de aplicación de estas técnicas no es una razón suficiente para explicar la falta de aplicación de las técnicas mentadas, sino que más bien habría que reparar en el hecho que los terapeutas conductuales "formados» emplean escasamente procedimientos que se derivan del paradigma conductista -una vez concluida su formación, en muchos casos, incluso prescinden por completo de ello. Se configura, de este modo, un conflicto entre el rechazo valórico espontáneo del modelo behaviorista del hombre, por un lado, y la abrumante evidencia empírica que apoya la efectividad terapéutica de los procedimientos generados sobre o a partir de esta visión-de-hombre, por el otro. De este modo, una salida facilista a este dilema podría consistir en prescindir de la aplicación de procedimientos que implican semejante imagen del hombre, demonificada y rechazada por su excesivo reduccionismo y su determinismo causalista.

2) Problemas de consolidación de una identidad terapéutica conductual

Las connotaciones valóricas e implicaciones éticas del modelo behaviorista del hombre es también la principal razón por la cual psicoterapeutas conductuales, a diferencia de otros terapeutas, suelen señalar que tienen grandes dificultades con asumir su identidad terapéutica. Antiguamente estas dificultades llevaban a que al interior de la heterogénea y dispareja comunidad terapéutica simplemente se silenciaba el hecho de que se trabajaba de manera cognitivo-conductual ocultando su credo y su práctica clínica ante los demás. O se enfatizaba que «en el fondo» uno tenía una formación clínica centrada en otras corrientes o escuelas (terapia gestáltica, familiar, de orientación analítica, etc.), desde las cuales se determinaba el fundamento -ecléctico- del actuar psicoterapéutico. En la actualidad, como apunta Westmeyer, a pesar de que la situación ha variado sensiblemente a lo largo de los últimos años, aún siguen existiendo considerables dificultades en la construcción de una identidad articulada y coherente, principalmente debido a la acción concertada de los aspectos señalados y la falta de historización discursiva de la corta pero convulsionada trayectoria de la terapia cognitivo-conductual. El recurso a la reconstrucción histórica de la secuencialidad de los enunciados y de la simultánea consideración de sus condiciones de posibilidad y de su contexto, en este lugar aparece como un recurso relevante y no lo suficientemente explotado. La identidad terapéutica, de este modo, pareciera ser menos una calidad sólida y preexistente, a la cual remiten los enunciados, sino, más bien, el resultado, siempre preliminar, de un permanente trabajo sobre la historia de las enunciaciones.

\section{Elementos para construir una posible respuesta al problema de las discrepancias teórico-prácticas generadas por la incompatibilidad de los diferentes supuestos antropológicos subyacentes en terapia cognitivo-conductual}

Los problemas mencionados, cuyo origen radica en las diferentes dificultades generadas a partir de las implicaciones y asociaciones derivadas de la imagen del hombre de la terapia conductual, contrariamente a lo que se suele pensar, no se relacionan necesariamente con el hecho de que (co)existan diferentes 
supuestos antropológicos preconceptuales en un mismo espacio discursivo. En cambio, las eventuales soluciones propuestas deberían apuntar a aprovechar esta aparente desventaja, a saber, la existencia simultánea de varios supuestos antropológicos, como un punto a su favor. Empero, la elaboración de una solución satisfactoria, aunque no necesariamente definitiva, requiere que se tomen en cuenta algunas consideraciones previas, que se introducirán a continuación de manera sumaria.

\section{1) Integración por renuncia a la exigencia o demanda de ubicuidad}

La condición necesaria más destacada para la formulación exitosa de una respuesta a los problemas anteriormente mencionados, a nuestro parecer, es una integración de los diferentes puntos de vista del hombre, postulados implícitamente. Los diversos supuestos antropológicos nucleares solamente podrán ser integrados si ambas direcciones, tanto la behaviorista como la cognitiva, abandonan su postura totalitaria y demandante, es decir, si están dispuestas a acotar y restringir su ámbito de validez. Una propuesta alternativa discutida en la actualidad (Fiedler 1996, Kazdin 1980) ha sido decidir sobre la base de resultados empíricos cuál concepción de hombre, cuál corriente teórica, puede ser aplicada en qué situación terapéutica para la descripción y comprensión de determinados cuadros clínicos. En caso de no existir datos científicos confiables, se opta por aquel procedimiento cuya concepción de hombre modela el paciente lo menos restrictiva y reductivamente posible.

Empero, esta solución, inspirada en los clásicos trabajos de Eysenck y Kazdin, destaca por su descomedido utilitarismo, su orientación reduccionista y su insuficiencia radical de elementos valóricos que permitan articular una dimensión ética conexa y coherente. La evidente falta de consistencia conceptual, su carácter fragmentado y escindido, falto de criterios racionales para la problematización adecuada de las dificultades y los conflictos producidos, junto a la orientación exclusivamente pragmática, favorecedora del credo del anything goes, característico de una versión secularizada del pensamiento postmoderno, son los principales argumentos que se pueden levantar en contra de esta tentativa de dar una respuesta al dilema anteriormente señalado.

2) Inserción del proceder terapéutico en una relación terapeuta-paciente basada en la horizontalidad

Por supuesto que no basta con el abandono de la exigencia de ubicuidad para resolver los embrollados problemas que aquejan a la terapia cognitivo-conductual hoy en día. Ello se debe principalmente a que siempre seguirían presentándose dilemas de difícil solución, como, por ejemplo, el problema de cómo actuar en tanto terapeuta conductual en el caso del tratamiento de la agorafobia. Gracias a las investigaciones de Fliegenbaum y Brengelmann, Faravelli y Albanesi, Ehlers y Margraf se sabe que el modelo behaviorista con los procedimientos de confrontación de los que dispone, posee los más altos índices de efectividad, pero, por el otro lado, la concepción de hombre implícita sigue siendo rechazada por la mayoría de los terapeutas por ser demasiado reduccionista. Frente a un caso de agorafobia se sugiere enfocar y problematizar explícitamente la relación terapeuta-paciente, una dimensión del tratamiento descuidada y olvidada por el conductismo ortodoxo. Éste, a excepción de los trabajos psicoanalíticos, ha sido una dimensión en extremo descuidada por la investigación en psicoterapia. Precisamente bajo la perspectiva del enfoque del selfmanagement (Kanfer, Reinecker y Schmelzer 2000) es de una importancia primordial concebir al paciente no únicamente como receptor pasivo de una determinada forma de terapia, armada y compuesta hasta en los últimos detalles por la figura omnipotente del terapeuta, sino de pensarlo como 
sujeto autónomo y actuante, que participa productivamente del proceso terapéutico. De esta manera, paciente y terapeuta determinan combinadamente el setting, las metas y los métodos que habrán de conducir hacia ellas, es decir, construyen entre los dos el marco y los parámetros de la terapia.

A diferencia de la verticalidad jerárquica característica de la concepción estandarizada de la relación terapeuta-paciente en el conductismo temprano, terapeuta y paciente en la actualidad son pensados en una especie de paralelismo estructural. Ambos son concebidos desde la misma perspectiva teórica, es decir, como entes reflexivos, dotados de ciertas facultades abstractas y propositivas a la vez, que les permiten la formación de una representación de sí mismos y del mundo circundante en el cual esta representación está inserta. Esta representación de sí mismo, al igual que la visión-de-mundo, Weltanschauung, resultante de la interpretación consensual e individual de la realidad, naturalmente que influye no solamente en su estructuración mental, sino que incide directamente en su comportamiento. La competencia de la formación de modelos (Gigerenzer) del paciente, condenada al olvido durante las décadas de dominio del paradigma behaviorista estricto, recién con el surgimiento del paradigma cognitivo encontró las condiciones histórico-culturales necesarias para su justa consideración. En este enfoque, terapeuta y paciente en el marco de un diálogo metacomunicativo sobre metas y caminos hacia estas metas se ponen de acuerdo dialécticamente acerca de las condiciones básicas que se han de cumplir para la planificación y puesta en marcha de la terapia. Mediante esta forma de configurar la relación terapéutica, el paciente de ser un objeto meramente pasivo, receptor paciente de intervenciones terapéuticas, se convierte en sujeto reflexivo, capaz de contribuir activamente al logro de sus metas terapéuticas.

Esta réplica a la situación problemática en la cual se encuentra entrampada la psicología cognitivoconductual en la actualidad, presenta la debilidad argumentativa que, al desplazar el acento sobre la reciprocidad y horizontalidad entre dos agentes equivalentes, se pierde de vista lo que tiene de específica la relación terapeuta-paciente, que se estructura de manera particular y diferenciada, diferente a cualquier otra relación interpersonal. El insistir en demasía en la correlatividad simétrica de la relación entre terapeuta y paciente significaría obviar de manera negligente los resultados palmarios y rotundos de años de investigación en psicoterapia (Grawe y Braun, Lambert y Bergin). Desarrollar in extenso este punto, a pesar de su innegable centralidad, nos llevaría más allá de los límites impuestos por este trabajo.

\section{3) Consideración de procesos inconscientes}

Al retratar la terapia cognitivo-conductual desde una perspectiva que se ha convenido en llamar "metateórica», se pueden identificar dos paradigmas fundamentales: por un lado, el paradigma behaviorista, que enfatiza las condiciones exteriores del comportamiento y -debido a su fundamentación histórica y epistemológica (Watson, Schorr)- tiene la tendencia de descuidar los procesos internos acompañantes. Por el otro lado, está el paradigma cognitivo, que coloca el énfasis justamente en dichos procesos intrapsíquicos, que transcurren «interiormente», con frecuencia al margen de la conciencia. Si el comportamiento humano, reconociendo así su complejidad irreductible, es visto desde una perspectiva integrativa, en opinión de Groeben, llama la atención la falta de teorías dedicadas explícitamente al análisis y la explicación de procesos inconscientes. A pesar de que, como postula Epstein, la mayoría del procesamiento de información en el hombre transcurre inconscientemente, entre las formulaciones teóricas asociadas al conductismo existen pocos proyectos consolidados que consideran y tematizan explícitamente estos procesos. Dicha tendencia a ignorar o descuidar la incidencia de procesos inconscientes se muestra, 
por ejemplo, en el hecho que entre los factores efectuales distinguidos por Grawe a propósito de sus investigaciones en terapia conductual -activación de recursos, actualización de problemas, ayuda activa para la superación de problemas, clarificación motivacional, sobre todo el factor clarificación motivacionalpresenta un déficit teórico relevante.

El desconocimiento radical de lo inconsciente desde luego que está relacionado con que la teoría paradigmática a la cual se suele acudir cuando de la comprensión de procesos inconscientes se trata, el psicoanálisis, contra el cual desde la posición conductual, como han demostrado Groeben y Herzog, existen reservas epistemológicas considerables. Al examinar la historia efectual del conductismo incluso se podría argumentar que éste se fundó en una oposición epistemológica hacia el psicoanálisis, definiéndose en sus comienzos justamente a través de este antagonismo, lo cual condicionó la exclusión y demonificación subsecuente de ciertos conceptos asociados tradicionalmente al psicoanálisis. Sin embargo, la terapia conductual contemporánea, emancipada de semejantes determinaciones históricas, debería superar estas posiciones ideologizadas y ya no cerrarse categóricamente a la exploración y consideración de procesos inconscientes, sobre todo en el ámbito de la investigación en psicoterapia. Han sido enumerados básicamente dos argumentos a favor de esta apertura cognitiva por parte del behaviorismo contemporáneo: primero, porque en psicología actualmente existen suficientes teorías científicas empíricamente respaldadas que describen y explican el actuar de procesos inconscientes (por ejemplo, la teoría de la disonancia y de la reactancia); segundo, porque justamente en el caso de las alteraciones psíquicas, con las cuales se las tiene que ver la psicoterapia, la recapacitación de procesos inconscientes suele jugar un rol fundamental, como, por ejemplo, en la conformación de relaciones interpersonales.

En consecuencia, si las terapias conductuales se dedicaran más intensamente al estudio de los procesos inconscientes, a los supuestos antropológicos centrales anteriormente expuestos se les podría agregar un tercer elemento, a saber, la perspectiva desde la cual la actividad humana puede ser enfocada como el resultado sobredeterminado de la confluencia de una serie de factores participantes, entre ellos, la incidencia de procesos inconscientes. Desde esta perspectiva, los productos del psiquismo remiten a una compleja y enrevesada historia de producción, en la cual convergen diferentes fuerzas, combinándose mediante diferentes formas de asociatividad. Recién entonces se puede pensar en la articulación de una concepción de hombre integrativa, capaz de describir, comprender y cambiar la totalidad de comportamientos humanos de manera diferenciada, éticamente comprometida.

\section{Discusión}

En la actualidad, la terapia conductual contemporánea, al considerar a los procesos mentales internos como una variable relevante para el tratamiento psicoterapéutico, ha experimentado un decisivo deslizamiento epistemológico desde su posición originaria, más ortodoxa, cimentada firmemente sobre postulados materialistas, hacia un punto de vista más flexible, abierto al debate con otras corrientes terapéuticas o incluso con otras disciplinas científicas. Este cambio de perspectiva, producto del devenir histórico propio de la terapia conductual, ha resaltado la necesidad de plantear con urgencia la pregunta por las posibilidades y las limitaciones asociadas a la integración de diversas modalidades discursivas y la consideración y articulación de diferentes resultados empíricos y teóricos.

Sin embargo, la incorporación de diversos elementos teóricos, que comúnmente se ve acompañada de la adopción de las más diversas herramientas terapéuticas, provenientes de otras escuelas y corrientes 
psicológicas, no ha de perder de vista el carácter único y distintivo de la terapia conductual. La evolución de la terapia conductual, a diferencia de lo que suele con otras escuelas, más conscientes de su procedencia y de sus orígenes, se ha caracterizado por su más bien escasa reflexión acerca de los procesos, vuelcos y giros de su paulatina maduración histórica. Dicha omisión no solamente ha llevado a que los terapeutas conductuales contemporáneos en su mayoría suelan desconocer sus antecedentes así como sus determinantes históricas, lo cual impide que formulen una posición diferenciada y crítica frente a estos, sino que asimismo han contribuido a crear una situación confusa y caótica, en la cual en un mismo espacio discursivo coexisten los más diferentes supuestos latentes acerca del hombre. Al no tematizar dicha discrepancia, se ha configurado una situación heterogénea, altamente fragmentada y carente de toda unidad, lo que contribuye al desconcierto y a la inseguridad de los terapeutas enfrentados con este dilema.

Al ser la psicología el discurso científico acerca de la mente y de los procesos mentales, la discusión de los supuestos antropológicos centrales, generalmente ignorados por los terapeutas clínicos, se vuelve un tema relevante tanto para la teoría y formulación de hipótesis como para la práctica efectiva. En el contexto de las teorías de sujeto más recientes, la revisión de los respectivos conceptos de hombre, derivados de los supuestos antropológicos implícitos, deviene una tarea fundamental para el presente. La revisión de la evolución de las respectivas representaciones de lo humano, promovidas por la terapia conductual, puede constituir un aporte significativo para la discusión y el debate acerca de sus posibles consecuencias terapéuticas. Un primer resultado preliminar sugiere que, como consecuencia de una comprensión de sí resueltamente modernista, desentendida de toda historicidad, afanosa en producir la ruptura tajante e irreversible con el pasado y la tradición del pensar, inspirada por el espíritu de comienzo radical, el racionalismo occidental se ha alienado de sus determinaciones y de sus raíces ilustradas, engendrando un concepto de hombre desterrado y reduccionista. Recién en los últimos decenios se ha modificado aquella concepción de hombre, deudora de una cruzada ideológica empeñada en "purificar» la ciencia objetiva de cualquier residuo humanista. Dado este giro en cuanto a la propuesta teórica identificada con el discurso conductual, el enfrentamiento crítico con la propia tradición del pensar bajo la perspectiva de los supuestos de hombre implicados puede constituir un aporte relevante para la integración de diversas demandas y diferentes desafíos para la práctica científica.

Naturalmente, la apertura hacia otros enfoques y otras modalidades de trabajo, si no quiere correr el peligro de sucumbir ante el eclecticismo y la adaptación al sentido común, ha de estar acompañada de un debate científico sólido y transparente acerca de los fundamentos epistemológicos subyacentes. Si se aspira a que estos se perfilen de manera clara y transparente, entonces la reflexión constante acerca de sus supuestos no tematizados es una tarea pendiente, que en ocasiones deberá recurrir, por ejemplo, a disciplinas emparentadas como la antropología filosófica y la filosofía de las ciencias. Lejos de obstaculizar y entorpecer la generación de nuevos conocimientos, dicho debate, en el cual los terapeutas del presente deben asumir un rol más protagónico, más activo, favorecerá la claridad y la consistencia de las respuestas encontradas a estas preguntas.

\section{Notas}

(1) El concepto de Menschenbildannahmen, que en este trabajo se traducirá como "supuestos antropológicos implícitos», se remonta principalmente a los trabajos de Gehlen, Plessner y von Weizsäcker. En el contexto de la problemática señalada no interesa tanto la reconstrucción pormenorizada de las etapas sucesivas de su historia efectual en el pensamiento académico especializado, sino más bien la delimitación y 
precisión de su respectiva repercusión en la discusión contemporánea en psicología. En la traducción que aquí se propone, una traducción que se reconoce como parcial y sesgada, se han omitido conscientemente las posibles asociaciones evocadas por el vocablo Bild, una expresión como consecuencia de la revalorización del lenguaje.

(2) Erróneamente, probablemente debido a la falta de sensibilidad y preocupación por la etimología y la historia efectual de los términos en cuestión, se ha tendido a hablar indistintamente de Menschenbild, imagen o figuración de hombre, por un lado, y concepto, Begriff, o representación, Vorstellung, de hombre, por el otro. Mientras que la primera palabra se remite a una tradición del pensar caracterizada por el empleo de una serie de metáforas visuales vinculadas a la hipóstasis concomitante de la razón (Foucault), el segundo vocablo se inscribe en el contexto histórico marcado por el auge del idealismo alemán y de la noción de representación, con lo cual estas teoretizaciones suelen encontrarse más próximas al pensamiento inaugurado por el linguistic turn, el pensamiento postmetafísico, la reformulación de la razón clásica y la inversión de la relación entre teoría y práctica (Habermas).

(3) Tanto en alemán como en español existen una serie de metáforas (be-greifen, aprehender, apresar, conquistar) vinculadas a la acción de comprender que destacan los aspectos activos, intervinientes, operativos de la comprensión y que desmienten su carácter receptivo, neutro y desapasionado.

(4) Desde la perspectiva de la historia de las ciencias parece interesante destacar el énfasis en el ambiente para el aprendizaje humano y el desarrollo del hombre. El examen de dicho factor externo posibilitó la investigación precisa de situaciones ambientales y su ulterior modificación.

(5) Una crítica fundada a la monopolización excesiva de las prácticas discursivas toleradas, avaladas y promovidas socialmente al interior de la comunidad lingüística se puede encontrar en Nägele y Strasser.

(6) Probablemente en vez de un enfoque, un término asociado en demasía al mundo representacional de la razón iluminadora, se debería hablar de un Ansatz, un punto de anclaje o de inserción, un tomar la palabra desde otro lugar.

\section{Bibliografía}

Bandura, A. 1986. Social foundations of thought and action: A social-cognitive theory. Engelwood Cliffs, New York: Prentice-Hall.

Barsch, A. 2000. Menschenbilder. Zur Pluralisierung der Vorstellung von der menschlichen Natur. Frankfurt a. M.: Suhrkamp.

Berger, P. y Luckmann, T. 1979. The social construction of reality: a treatise in the sociology of knowledge. Harmondsworth: Penguin.

Faller, H., Wagner, R. F. \& Weiß, H. 2000. Therapeutische Orientierung und Wahrnehmung des Patienten bei der Erstuntersuchung. Ein empirischer Vergleich von tiefenpsychologisch/analytisch und kognitivverhaltenstherapeutischen Therapeuten. Psychotherapeut 45: 108-115.

Feldman, M. P. y McCulloch, M. J. 1971. Homosexual behavior. Therapy and assessment. Oxford: Pergamon Press.

Fiedler, P. 1996. Verhaltenstherapie in und mit Gruppen. Weinheim: PVU Beltz. 
Fiedler, P. 1997. Die Verhaltenstherapie zu Beginn der 90er Jahre. Historische Entwicklung und Perspektiven. En: Zielke, M., \& Sturm, J. (ed.). Handbuch Stationäre Verhaltenstherapie. Weinheim: PVU Beltz, pp. 33-41.

Groeben, N. 1986. Handeln, Tun, Verhalten als Einheiten einer verstehend-erklärenden Psychologie. Tübingen: Francke.

Habermas, J. 1997. Theorie des kommunikativen Handelns. 2 tomos, Frankfurt a. M.: Suhrkamp.

Kanfer, F. H. 1970. Self-regulation: research, issues, and speculations. En: Neuringer, C. y Michael, J. L. (ed.). Behavior modification in clinical psychology. New York: Apleton-Century-Croft, pp. 178-220.

Kanfer, F. H. 1979. Self-management: strategies and tactics. En: Goldstein, A. P. y Kanfer, F. H. (ed.). Maximizing treatment gains: Transfer enhancement in psychotherapy. New York: Academic Press, pp. 185224.

Kanfer, F. H., Reinecker, H. y Schmelzer, D. 2000. Selbstmanagement-Therapie. Ein Lehrbuch für die klinische Praxis (3a edición). Heidelberg: Springer.

Kazdin, A. E. 1978. History of behavior modification. Baltimore: University Park Press.

Kazdin, A. E. 1980. Reserach design in clinical psychology. Needham Heights MA: Ayllyn \& Bacon.

Lieb, H. y Lutz, R. 1992. Verhaltenstherapie : ihre Entwicklung - ihr Menschenbild. Göttingen; Stuttgart: Verlag für Angewandte Psychologie.

Mahoney, M. J. 1997. Kognitive Verhaltenstherapie. Neue Entwicklungen und Integrationsschritte. München: Pfeiffer.

Margraf, J. y Schneider, S. 2000. Paniksyndrom und Agoraphobie. En: Margraf, J. (ed.). Lehrbuch der Verhaltenstherapie (2a edición). tomo 2. Berlin: Springer, pp- 1-27.

Margraf, J. (2000). Grundprinzipien und historische Entwicklung. En Margraf, J. (ed.). Lehrbuch der Verhaltenstherapie (2a edición). Tomo 1. Berlin: Springer, pp. 1-30.

Reinecker, H. 1998. Zwänge. Diagnose, Theorien, Behandlung._Bern: Huber.

Schorr, A. 1984. Die Verhaltenstherapie. Ihre Geschichte von den Anfängen bis zur Gegenwart. Weinheim: Beltz.

Westmeyer, H. (1984). Von den Schwierigkeiten, ein Behaviorist zu sein, oder Auf der Suche nach einer behavioristischen Identität. En Lenk, H. (ed.). Handlungstheorien - interdisziplinär. Tomo 3. München: Fink, 179-200.

Wittchen, H.-U. 1996. Klinische Psychologie und Verhaltenstherapie - zwischen Aufstieg und Erosion. Verhaltenstherapie 6: 170-177.

Recibido el 9 abril de 2012

Aceptado el 25 de julio de 2012 